\title{
A guide to physiotherapy in urogynecology for patient care during the COVID-19 pandemic
}

\author{
Cristine Homsi Jorge Ferreira ${ }^{1}$ (D) - Patricia Driusso ${ }^{2} \cdot$ Jorge Milhem Haddad $^{3} \cdot$ Simone Botelho Pereira $^{4,5}$. \\ Ana Carolina Nociti Lopes Fernandes ${ }^{1} \cdot$ Debora Porto $^{6} \cdot$ Bianca Manzan Reis $^{2} \cdot$ Lilian Rose Mascarenhas ${ }^{7}$. \\ Luiz Gustavo Oliveira Brito ${ }^{8}$. Elizabeth Alves Gonçalves Ferreira ${ }^{6}$
}

Received: 16 July 2020 / Accepted: 14 September 2020 / Published online: 28 September 2020

(C) The International Urogynecological Association 2020

\begin{abstract}
Introduction and aim Physiotherapy in urogynecology faces challenges to safely continuing its work, considering the adoption of social distancing measures during the COVID-19 pandemic. Some guidelines have already been published for urogynecology; however, no specific documents have been produced on physiotherapy in urogynecology. This article aimed to offer guidance regarding physiotherapy in urogynecology during the COVID-19 pandemic.

Methods A group of experts in physiotherapy in women's health performed a literature search in the Pubmed, PEDro, Web of Science and Embase databases and proposed a clinical guideline for physiotherapy management of urogynecological disorders during the COVID-19 pandemic. This document was reviewed by other physiotherapists and a multidisciplinary panel, which analyzed the suggested topics and reached consensus. The recommendations were grouped according to their similarities and allocated into categories.

Results Four categories of recommendations (ethics and regulation issues, assessment of pelvic floor muscle function and dysfunction, health education and return to in-person care) were proposed. Telephysiotherapy and situations that need inperson care were also discussed. Regionalization is another topic that was considered.

Conclusion This study provides some guidance for continuity of the physiotherapist's work in urogynecology during the COVID-19 pandemic, considering the World Health Organization recommendations and the epidemiological public health situation of each region. Telephysiotherapy can also be used to provide continuity of the care in this area during the COVID19 pandemic, opening new perspectives for physiotherapy in urogynecology.
\end{abstract}

Keywords COVID-19 $\cdot$ Pandemic $\cdot$ Physiotherapy $\cdot$ Rehabilitation $\cdot$ Pelvic floor muscles $\cdot$ Urogynecology

Cristine Homsi Jorge Ferreira

cristine@ fmrp.usp.br

1 Pelvic Floor Function Laboratory, Department of Health Sciences, Ribeirão Preto Medical School, University of São Paulo (USP), Ribeirão Preto, SP, Brazil

2 Women's Health Research Laboratory, Physical Therapy Department, Federal University of São Carlos (UFSCar), São Carlos, SP, Brazil

3 Department of Obstetrics and Gynecology, Urogynecology Section, University of São Paulo (USP), São Paulo, SP, Brazil

4 Department of Surgery, School of Medical Sciences, Postgraduate Program in Surgery Sciences, State University of Campinas (UNICAMP), Campinas, SP, Brazil
5 Motor Science Institute, Post-Graduate Program in Rehabilitation Sciences, Federal University of Alfenas (UNIFAL-MG), Alfenas, MG, Brazil

6 Physiotherapy in Women's Health Research Laboratory, Department of Physiotherapy, Communication Sciences \& Disorders and Occupational Therapy, University of São Paulo (USP), São Paulo, SP, Brazil

7 Department of Human Movement Sciences, State University of Pará (c), Belém, PA, Brazil

8 Department of Obstetrics and Gynecology, School of Medical Sciences, University of Campinas (UNICAMP), Campinas, SP, Brazil 


\section{Introduction}

The COVID-19 pandemic has changed all health care scenarios, and many institutions have interrupted patient care. Women's health physiotherapy focused on urogynecology has been almost completely interrupted, and thousands of patients have been left unattended. If we consider the number of patients that would have needed care, we can find data indicating that about $25 \%$ of women have symptomatic PFDs [1]. Although the problems treated are generally not urgent, the level of discomfort can be severe, creating a negative impact on quality of life. Social distancing during the pandemic could increase PFD-related suffering and other morbidities affecting women's quality of life because of multiple factors such as increased obesity, physical inactivity, stress and difficulty having access to safe and comprehensive health care, including physiotherapy $[2,3]$. An early initiation of the rehabilitation process in urogynecology is considered a crucial factor for women's health [4].

Physiotherapy is a profession that focuses on human movement and functionality [5], basing its interventions on thorough evaluation of general aspects regarding symptoms and specific aspects related to pelvic floor muscle (PFM) function and dysfunctions in the field of women's health. Telehealth services can facilitate public health strategies during the pandemic, offering guidance to patients while preserving social distancing [6]. According to the World Confederation for Physical Therapy, "Digital Practice is a term used to describe health care services, support and information provided remotely via digital communication and devices" [7]. It includes telephone calls, platforms for video calls, video recordings and other forms of contact with technological assistance. The term adopted throughout this manuscript to refer to the practice of digital physiotherapy is telephysiotherapy, although other terms such as virtual physiotherapy can be found in the literature.

A number of randomized controlled trials (RCTs) have tested the efficacy of telephysiotherapy for some health conditions [7]. However, PFD in-person physiotherapy treatment involves some specificities, such as dealing with intimate issues, patients' embarrassment regarding their symptoms and the need to perform a pelvic floor muscle examination. These specificities make privacy and ethical issues in this area especially relevant, as there are limitations and concerns when considering telephysiotherapy in urogynecology. Nonetheless, telephysiotherapy could provide safe continuity to physiotherapy care in urogynecology. Despite this, there are only a few studies addressing telephysiotherapy in the treatment of PFDs, and they present promising results $[8,9]$.

Although Grimes et al. [4] published excellent guidelines for treating urogynecological patients during the pandemic, their article was not specifically focused on physiotherapy. Therefore, this article aims to offer specific guidance regarding physiotherapy in urogynecology during the COVID-19 pandemic.

\section{Methods}

\section{Study design}

This was a descriptive study proposing guidance and recommendations based on a literature review and a group of experts' opinions about the topic.

\section{Development of the recommendations: Data search and analysis}

This document was coordinated by a group of experts who are members of the Brazilian Association of Physiotherapy in Women's Health (Associação Brasileira de Fisioterapia em Saúde da Mulher - ABRAFISM). It included representative consultants from the Brazilian Association of Urogynecology and the Pelvic Floor (Associação de Uroginecologia e Assoalho Pélvico - UROGINAP) and the Latin American Association of the Pelvic Floor (Associación Latino Americana de Piso Pelvico-ALAPP). The process of discussing and writing the document followed a three-step modified Delphi method and took place in June and July 2020.

The first step was a literature review, conducted from June 9 to July 11, 2020, in the following databases: Pubmed, PEDro, Web of Science and Embase. All searches were restricted to the English and Portuguese languages. For all topics, we sought existing systematic reviews and primary studies. We also consulted the guidelines of international associations, such as the World Confederation for Physical Therapy (WCPT), International Urogynecological Association (IUGA) and International Continence Society (ICS). Two independent reviewers (ACNLF and BMR) performed the selection process for primary studies by reviewing titles, abstracts and reading full texts, based on the previously described proposed inclusion criteria. The reference lists of included studies were screened independently to identify possible studies not retrieved by the electronic search. Any disagreements during the selection process were resolved by discussion until a consensus was reached by the group of specialists.

The terms used in the search strategy in the Pubmed, Web of Science and Embase databases were: (physiotherapy* OR "physical therapy*" OR rehabilitation) AND ("pelvic floor dysfunctions" OR "pelvic floor symptoms" OR "pelvic floor" OR "pelvic floor muscle" OR "lower urinary tract symptoms" OR "urinary incontinence" or "urinary leakage" OR "anal incontinence" OR constipation OR "fecal incontinence" OR "bowel symptoms" OR incontinence OR "pelvic organ 
prolapse" OR "rectal prolapse" OR "vaginal prolapse" OR "pelvic prolapse" OR "sexual dysfunction" OR vaginismus OR dyspareunia OR "pelvic pain") AND ("mobile health*" OR e-health OR "mobile app*" OR "digital health*" OR telemedicine OR "tele rehabilitation" OR telehealth OR "telephone-delivered care" OR "telephone health*" OR "home treatment" OR "home-based" OR covid-19 OR "covid 19" OR covid OR coronavirus).

The strategy for the advanced search in PEDro was limited by anatomical regions (perineum or genito-urinary system) and discipline (continence and women's health). The following terms were used in "Abstract \& Title": covid, coronavirus, e-health, mobile app, mobile health, digital health, telemedicine, tele rehabilitation, tele health, telephone-delivered care, telephone health and home-based treatment. One search was generated for each term.

The second step was performed by a group of experts that discussed issues related to the digital practice of physiotherapy in the treatment of PFD, while another group read the identified manuscripts and guidelines related to the topic. The first group was composed of two women's health physiotherapists (CHJF and LRM) and current members of the executive board of ABRAFISM. Both professionals have > 20 years of experience working in the area as clinicians and researchers. Furthermore, two women's health physiotherapists (EAGF and PD) with extensive experience in the field complemented the initial draft with additional modifications. The second group was composed of three clinical physiotherapists with experience of between 2 and 5 years working in the area, who participated in the preparation of the document. In this step, the experts had several online meetings to discuss the findings and vote for the recommendations, with careful attention to the items that presented a disagreement rate $>50 \%$.

The third step involved a final meeting to thoroughly revise the document and propose the final recommendations. In this step, after consensus was reached, the recommendations were grouped according to their similarities and assigned to categories. Subsequently, a group of consultants, including two urogynecologists, members of the Executive Board of UROGINAP (JH and LGOB) and IUGA (International Urogynecological Association), and a physiotherapist specializing in women's health and representing ALAPP (SB), made additional contributions and approved the recommendations. Due to the COVID-19 pandemic, no face-to-face meetings were scheduled.

\section{Results}

Finally, expert consensus was used to create and summarize the following topics: (1) recommendations about the continuity of physiotherapy care during the COVID-19 pandemic, ethics and regulation issues; (2) recommendations on physiotherapeutic assessment of PFD symptoms and PFM function using telephysiotherapy; (3) health education recommendations and specific recommendations about physiotherapeutic interventions for women with PFD during the pandemic; (4) returning to in-person clinical care.

The electronic search returned a total of 257 references and, after the selection process, 49 studies were included in the final analysis (Fig. 1).

The guidelines proposed four categories of recommendations, as presented below:

1. Recommendations about the continuity of physiotherapy care during the COVID-19 pandemic, ethics and regulation issues.

- To provide continuity of care using digital physiotherapy modalities whenever possible. Remote monitoring should be considered, especially for women who were already undergoing physiotherapy treatment of urogynecology and proctological symptoms [10, 11].

- To discuss with patients the feasibility of performing physiotherapeutic care using digital physiotherapy.

- To observe all norms and rules contained in the Codes of Ethics and Deontology of Physiotherapy and take into consideration that this may be influenced by each country's specifics as well as specific regulations and/ or standards of service involved and possible new regulations related to the pandemic, even when using digital physiotherapy [12].

- To consider cultural aspects, technological and personal limitations on the use of technology, patient availability and access to technology, and familiarity of physiotherapists and patients with technological tools [13].

- To choose the most appropriate digital method, considering the specific case to be treated as well as patient access and preferences.

- To be more cautious about privacy of care and storage of patient data. The registration of information and therapeutic exercises must be maintained with extra care, considering the vulnerability of some digital platforms [14].

- To ensure patient privacy, information accuracy, security and confidentiality. Information about the benefits and disadvantages of online care should be made clear to patients [12].

- To share written guidelines with patients prior to or after the session [15].

- To comply with privacy and ethics regulations when using any photos of the patient for assessment purposes [16].

- To inform the patient in advance when recording remote physiotherapy sessions and when recording 
Fig. 1 PRISMA flow diagram of study selection process

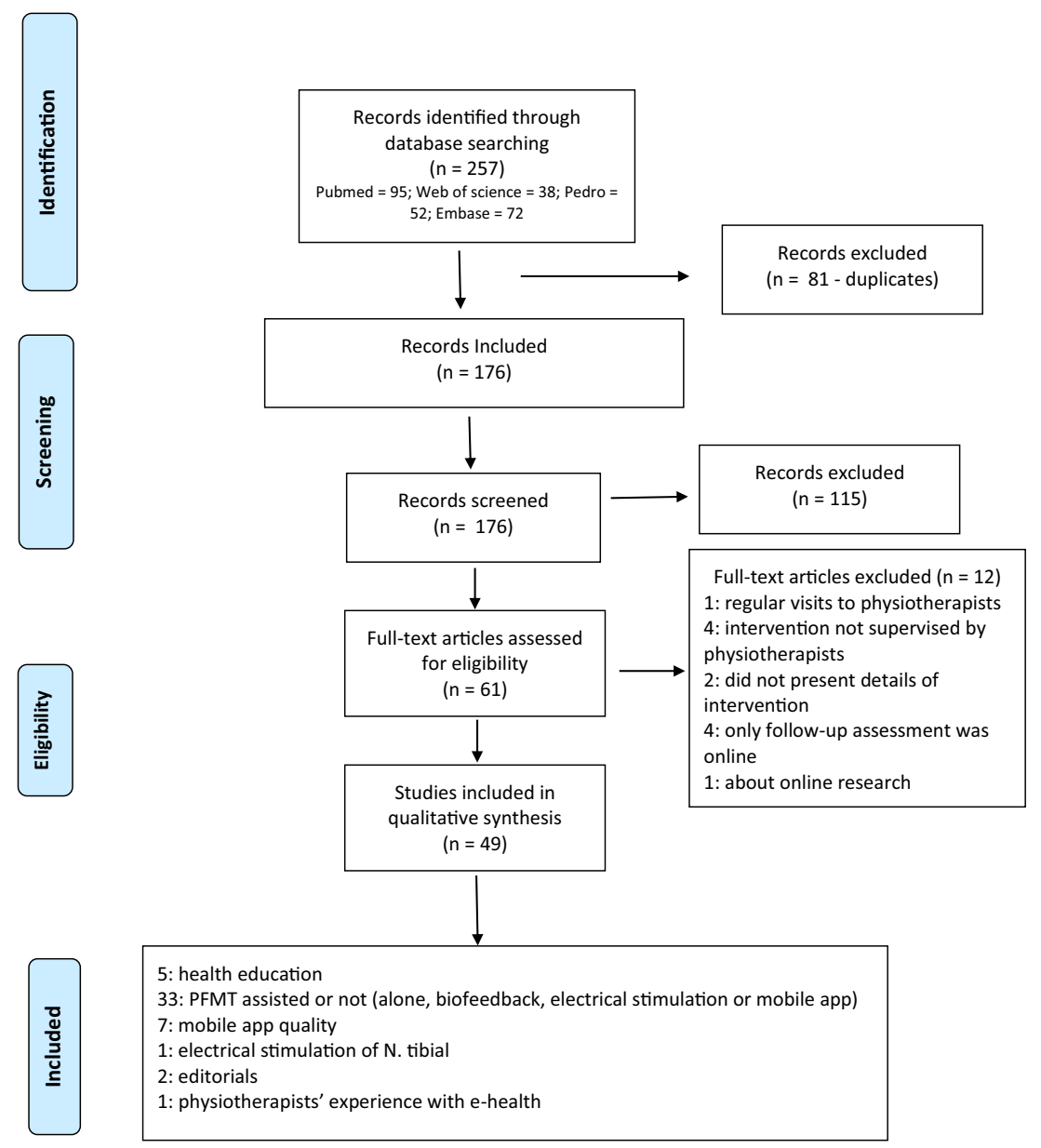

videos of the body and the posture in general for movement analysis.

- To align patient expectations with services and be clear in establishing the specific goals, outcomes and indicators for each virtual meeting, so that the patient also feels in control of their health and involved with the treatment [10].

2. Recommendations on physiotherapeutic assessment of PFD symptoms and PFM function using telehealth.

- History should be taken with a focus on: obtaining as many details as possible about pelvic floor functionality, adding to the voiding diary and validating questionnaires to assess pelvic floor symptom severity and impact on quality of life [17].

- Voiding diary apps can be recommended to women who are willing to use them. The quality of apps should be considered when choosing them as well as translation and validation into the languages to be used [18].

- Attempts to guide complete and accurate remote PFM assessment in clinical practice should be avoided because of the lack of evidence at this time and the impossibility of assessing tonus, strength and endurance of the pelvic floor without an internal examination.

- It is important to avoid requesting images and videos involving exposure of women's bodies for remote synchronous or asynchronous evaluation unless their consent is obtained and they are clearly informed about all the limitations and uncertainty regarding the accuracy of the evaluation and the privacy risks involved.

- Information about women's capacity to contract their PFMs can be investigated using alternative methods such as the stop test. It can be explained to women and used cautiously as a means for them to self-assess their PFM contraction capacity as well as the use of terms that can facilitate their capacity to perceive their PFM contraction [17, 19].

- The stop test should not be used routinely to perform pelvic floor muscle training (PFMT) [20].

- Instructions for self-palpation can be given to women who culturally accept it, and they should be told to perform it in a private environment and that it should be avoided during real-time video calls $[17,19]$. 
- Self-estimation of women's ability to contract their PFMs seems to be poor, especially in women with weak PFM contraction, meaning that the results should be interpreted with caution [21].

3. Health education recommendations and specific recommendations about physiotherapeutic interventions for women with PFD symptoms during the pandemic

\section{Health education recommendations}

- To instruct women about general health issues and their influence on PFD using different types of illustrative resources.

- To give detailed information about the importance and benefits of physical exercise for weight control, healthy diet, appropriate water intake and prevention of constipation.

- To provide women with general self-perception, stretching and relaxation exercises and strategies, considering the anxiety issues involved in this time of the pandemic and their need for strategies to promote their health and well-being.

- To counsel women about the use of protectors (absorbents, diapers) and hygienic issues.

- To provide women with essential information about pelvic floor function and dysfunction and options for treatment prior to implementing physiotherapy and to educate the general community in order to increase women's knowledge about these topics [22].

- To use educational illustrations, videos and other resources that can facilitate women's comprehension of pelvic floor contraction.

- To consider possible adherence modifiers while conducting health education aiming to improve treatment adherence. The use of health behavior theories is suggested.

\section{Recommendations about specific physiotherapy interventions for women with PFD}

- Women who are able to contract their PFMs should be advised to start or continue their PFMT program, considering the same parameters prescribed for them based on exercise physiology principles (frequency, intensity and duration).

- Whenever possible, sessions should be remotely supervised by the physiotherapist, using synchronic video calls to answer questions, clear up any possible doubts for patients and reinforce instructions for proper exercises [23].
- If women have limited or no internet access, adherence can be monitored using telephone calls, and motivation could be provided by sending written instructions using e-visits (e-mails).

- Different options to facilitate PFMT adherence,such as training diaries, phone calls, apps and others, should be indicated based on women's cultural and socioeconomic background, especially when supervised training is not possible.

- The use of apps can be an adjunct method for the physiotherapeutic treatment prescribed, based on physiotherapy assessment of patients' profiles. Consider patient access, familiarity with the use of technologies, autonomy, level of education and, especially, how much patients like and feel confident in the use of technology.

- The quality of PFMT apps should be considered when making recommendations [24] and should be discussed with women for shared clinical decision-making, considering clinical evaluation and physiotherapeutic objectives [25].

- Physiotherapists must know the functionalities of the many paid and unpaid apps to be able to guide women throughout the entire therapeutic process.

- In recommending the use of apps, consider the possibility of performing data extraction, their educational features, availability of reinforcements/reminder systems, self-monitoring, whether the app is free on download platforms and the cost benefit for patients to acquire an app, especially considering their socioeconomic status $[25,26]$.

- Consult RCTs and the systematic reviews on the topic that investigated different mobile technologies and apps for urinary incontinence. One systematic review concluded there is level 2 evidence of benefits in using mobile technologies to improve UI, patient satisfaction, adherence and costs [27]. Another systematic review included three RCTs of low to moderate quality and concluded that mobile apps reduced urinary symptoms [28].

- Give preference to the use of well-evaluated apps according to validated scales for assessing app quality and with demonstrated effectiveness in RCTs of good methodological quality [25].

- Women who are not able to contract their PFMs need to be informed about the limitations of using all physiotherapeutic resources when receiving telephysiotherapy. The remote options available should be discussed, including giving instructions exclusively using analogies such as videos simulating PFM contraction, self-visualization, self-palpation and the stop test.

- Prescriptions and instructions for home self-applied electrostimulation, biofeedback and vaginal cones should be carefully analyzed, considering the low level of evidence showing risks and benefits [16, 26]. 
- Before recommending the use of any probes or equipment for home use, physiotherapists must pay attention to possible required registration with regulatory agencies in their countries. In addition, clear instructions must be given about issues around cleaning and preventing infection.

- Bladder training and behavioral treatment should be provided and supervised by physiotherapists, including any types of intervention to delay urination, programmed urination and patient education to modify urination habits.

4. Recommendations for returning to in-person clinical care.

- To return to in-person clinical care according to the government regulations of each country.

- To perform any care, regardless of the case, with surgical masks and gloves. It is also possible to add the use of face shields or glasses.

- To perform pre-care phone screening and ask about possible respiratory symptoms.

- To confirm that patients have obtained negative results for COVID-19 testing before scheduling. This should be reinforced in cases of suspected infection or if patients have been in contact with positive COVID19 cases.

- To perform cleaning and disinfection of the place of care and objects used between sessions for different patients, setting aside specific time for this.

- To not share the use of pens, clipboards or telephones with patients.

- To measure patients' temperature before starting sessions.

- To provide materials and guidelines for hygiene procedures to be performed in the service environment, such as $70 \%$ alcohol, disposable tissues and appropriate facilities for handwashing.

- To perform a correct disposal of materials used during services.

- To carefully assess patient clinical performance status, especially for patients recovering from COVID19 , because of the effect of the disease on the musculoskeletal and respiratory systems. Symptoms such as myalgia, decreased breathing capacity, coughing and fatigue, anosmia and dyspnea are important signs of worsening of the disease.

\section{Discussion}

This article offers a list of recommendations for practicing physiotherapy in the treatment of urogynecological conditions throughout the COVID-19 pandemic. The most important recommendation is to, as much as possible, offer continuity of physiotherapy care, using some type of remote service. Although this is already part of physiotherapy practice in some places, it might be limited because of physiotherapy regulations or restrictions on internet access and technology in many countries.

At this time, the most important thing seems to be the possibility of making contact with patients using whatever remote technology enables physiotherapists to get information about patients' situations and monitor implemented therapies. In many places, the only way to do this is by telephone. Another option that should be considered is the use of free platforms for video calls, taking into consideration local physiotherapy regulations and privacy and ethical issues. Ethical issues should be looked at very carefully, considering that physiotherapy in women's health deals with sensitive issues that can lead to patients' embarrassment if there are breaches of confidentiality.

Despite the many challenges, the literature has described many advantages of telephysiotherapy, including encouragement of self-management, increases in flexibility for healthcare delivery and decreases in sick-leave duration [29]. Other possible advantages include reduced costs to transport patients and physiotherapists to health services, a possible increased number of sessions if necessary, time saved for patients waiting to be treated and being able to assist patients from distant locations. It is an opportunity for physiotherapists to work creatively using technology, valuing the evaluation of aspects of the patients' history and habits, communication skills and provision of instructions to help them change life habits and adhere to treatment $[5,29]$.

Furthermore, the accuracy of assessment tests and efficacy of treatment have been investigated and confirmed in different areas of physiotherapy [6]. However, only a few studies have investigated the efficacy of telephysiotherapy in the treatment of PFD. Although small RCTs have shown promising results for some remote interventions, such as home PFMT and lifestyle interventions, there is not strong evidence about the efficacy of this format for treatment. A few small studies on the use of home electrostimulation and biofeedback to treat urinary and fecal incontinence in women have achieved good results, but they present high risk of bias, and the patients had previously been trained to self-use the devices [26]. Additionally, the devices have established protocols, allowing patients to manipulate only current intensity. It is essential for physiotherapists to know patients' profiles and include them in the clinical decision-making process and to balance the cost-effectiveness of treatments.

The role of physiotherapists includes more than the use of therapeutic resources, such as electrical stimulation and manual therapy; it also involves aspects related to interactions with patients, communication skills, and selection and use of combined therapeutic resources based on the physiotherapist's assessment of patients. All these comprehensive aspects have 
the potential to impact the results of telephysiotherapy compared to in-person sessions. Future pragmatic RCTs should further investigate the efficacy of telephysiotherapy in the treatment of uroginecological problems, since the literature related to this topic is still scarce.

Pelvic floor muscle training is the first-line treatment for non-neurogenic UI and mild and moderate pelvic organ prolapse [30]. There are many remote synchronous and asynchronous ways for physiotherapists to prescribe and monitor individualized digital sessions of PFMT. However, it does not seem to currently be possible to accurately and completely carry out PFM assessment using digital technologies. The most critical limitation when using telephysiotherapy is assessment of women's capacity to contract their PFMs. Even with this limitation, PFMT should be prescribed, since at least a proportion of women who are not able to contract their PFMs in the first assessment will acquire this ability by receiving proper instructions on how to do so exclusively in this format [31]. When considering the use of the stop test or any devices, such as vaginal cones and all types of home biofeedback, physiotherapists must provide very clear instructions to patients regarding proper use and safety issues. Patients should be informed that performing PFMT by stopping the urine stream is not recommended, because it can potentially disturb the neurophysiological bladder voiding mechanism and increase the risk of urinary infection [20].

The return to in-person physiotherapy will require additional care to protect physiotherapists and patients. This article offered some guidance related to this issue. However, local epidemiological situations and sustained declines in the number of COVID-19 cases must be taken into account for a safe return of face-to-face care.

Some limitations of these guidelines must be highlighted, starting with the short time during which the study was carried out and the absence of specific studies on the topic. This led to recommendations based on expert opinion, combined with no or low levels of evidence. However, it was possible to offer some guidance to professionals working in the area and also indicate some directions for future research on telephysiotherapy in urogynecology.

Telephysiotherapy will not be a feasible option for all patients, but it provides opportunities to offer continuity of care to at least some patients and to advance knowledge and clinical practice in this field. The challenges imposed by the pandemic may lead to greater appreciation of the communicative and educational abilities of physiotherapists.

The present study provided some guidance for continuity of the work of physiotherapists in urogynecology during the COVID-19 pandemic, considering the World Health Organization recommendations and the epidemiological public health situation of each region. Telephysiotherapy is an option for continuity of care during the pandemic, especially considering that education is a large component in physiotherapy, opening new perspectives for the development of practice and research in urogynecology and pelvic health.

Funding Coordenação de Aperfeiçoamento de Pessoal de Nível Superior - (CAPES)-Finance Code 001.

\section{Conflict of interest None.}

Disclosures The authors completed the ICJME Form for Disclosure of Potential Conflicts of Interest and reported no conflicts of interest.

Ethics approval Not applied.

\section{References}

1. Nygaard I, Barber MD, Burgio KL, et al. Prevalence of symptomatic pelvic floor disorders in US women. JAMA. 2008;300(11): 1311-6. https://doi.org/10.1001/jama.300.11.1311.

2. Flegal K, Carroll MD, Brian K, et al. Association of all-cause mortality with overweight and obesity using standard body mass index categories. J Am Med Assoc. 2013;309(1):71-82. https://doi.org/ 10.1001/jama.2012.113905.

3. Ekelund U, Tarp J, Steene-Johannessen J, et al. Dose-response associations between accelerometry measured physical activity and sedentary time and all cause mortality: systematic review and harmonised meta-analysis. BMJ. 2019;366:14570. https://doi.org/ 10.1136/bmj.14570.

4. Grimes CL, Balk EM, Crisp CC, Antosh DD, Murphy M, Halder $\mathrm{GE}$, et al. A guide for urogynecologic patient care utilizing telemedicine during the COVID-19 pandemic: review of existing evidence. Int Urogynecol J. 2020;31(6):1063-89. https://doi.org/10.1007/ s00192-020-04314-4.

5. Online document World Physiotherapy https://world.physio/ resources/what-is-physiotherapy. Accessed 12 July 2020.

6. O'Connor M, Asdornwised U, Dempsey ML, Huffenberger A, Jost $\mathrm{S}$, Flynn D, et al. Using telehealth to reduce all-cause 30-day hospital readmissions among heart failure patients receiving skilled home health services. Appl Clin Inf. 2016;7(02):238-47. https:// doi.org/10.4338/ACI-2015-11-SOA-0157.

7. Lee A, Finnin K, Holdsworth L, Millette D, Peterson C. Report of the WCPT/INPTRA Digital physical therapy practice task force. WCPT/INPTRA Digital Practice Final Report vs.7 15 May 2019.

8. Lindh A, Sjöström M, Stenlund H, Samuelsson E. Non-face-to-face treatment of stress urinary incontinence: predictors of success after 1 year. Int Urogynecol J. 2016;27(12):1857-65. https://doi.org/10. 1007/s00192-016-3050-4.

9. Sjöström M, Umefjord G, Stenlund H, Carlbring P, Andersson G, Samuelsson E. Internet-based treatment of stress urinary incontinence: a randomised controlled study with focus on pelvic floor muscle training. BJU Int. 2013;112(3):362-72. https://doi.org/10. 1111/j.1464-410X.2012.11713.x.

10. Firet L, de Bree C, Verhoeks CM, Teunissen DA, Lagro-Janssen AL. Mixed feelings: general practitioners' attitudes towards eHealth for stress urinary incontinence-a qualitative study. BMC Fam Pract. 2019;20(1):21. https://doi.org/10.1186/s12875-0190907-x.

11. Brusciano L, Gualtieri G, Gambardella C, Tolone S, Lucido FS, del Genio $\mathrm{G}$, et al. When preserving life becomes imperative, quality of life is eclipsed! COVID-19 outbreak impacting patients with pelvic floor disorders undergoing pelvic floor rehabilitation. BJS. 2020;107(8):e242e242. https://doi.org/10.1002/bjs.11675. 
12. Kaplan B, Litewka S. Ethical Challenges of Telemedicine and Telehealth. Camb Q Healthc Ethics. 2008;17(4):401-16 https:// www.cambridge.org/core/journals/cambridge-quarterly-ofhealthcare-ethics/article/ethical-challenges-of-telemedicine-andtelehealth/5D777B4EDE6E97934FB4B442F9CE0B33.

13. Lee DD, Arya LA, Andy UU, Sammel MD, Harvie HS. Willingness of women with pelvic floor disorders to use mobile technology to communicate with their health care providers. Female Pelvic Med Reconstr Surg. 2019;25(2):134-8. https://doi. org/10.1097/SPV.0000000000000668.

14. Tiffin N, George A, LeFevre AE. How to use relevant data for maximal benefit with minimal risk: digital health data governance to protect vulnerable populations in low- income and middleincome countries. BMJ Glob Health. 2019;4(2):e001395 https:// gh.bmj.com/content/4/2/e001395.

15. Dumoulin C, Cacciari LP, Hay-Smith EJC. Pelvic floor muscle training versus no treatment, or inactive control treatments, for urinary incontinence in women. Cochrane Database Syst Rev. 2018;10:CD005654. https://doi.org/10.1002/14651858. CD005654.pub4.

16. Bokne K, Sjöström M, Samuelsson E. Self-management of stress urinary incontinence: effectiveness of two treatment programmes focused on pelvic floor muscle training, one booklet and one internet-based. Scand J Prim Health Care. 2019;37(3):380-7. https://doi. org/10.1080/02813432.2019.1640921.

17. Woodley SJ, Boyle R, Cody JD, Mørkved S, Hay-Smith EJC. Pelvic floor muscle training for prevention and treatment of urinary and faecal incontinence in antenatal and postnatal women. Cochrane Database Syst Rev. 2017;12:CD007471. https://doi.org/ 10.1002/14651858.CD007471.pub3.

18. Vaccari NA, da Silveira LTY, Bortolini MAT, Haddad JM, Baracat EC, Ferreira EAG. Content and functionality features of voiding diary applications for mobile devices in Brazil: a descriptive analysis. Int Urogynecol J. 2020. https://doi.org/10.1007/s00192-02004382-6.

19. Mateus-Vasconcelos ECL, Ribeiro AM, Antônio FI, Brito LG de $\mathrm{O}$, Ferreira CHJ. Physiotherapy methods to facilitate pelvic floor muscle contraction: a systematic review. Physiother Theory Pract. 2018;34(6):420-32. https://doi.org/10.1080/09593985.2017. 1419520.

20. Bø K, Morkved S. Chapter 6: pelvic floor and exercise science: motor learning. In: Bø K, Berghmans B, Morkved S, van Kampen M, editors. Evidence-based physical therapy for the pelvic floor. 2nd ed. Elsevier: Philadelphia; 2015. p. 111-6. https://www. elsevier.com/books/evidence-based-physical-therapy-for-thepelvic-floor/bo/978-0-7020-4443-4.

21. Uechi N, Fernandes ACNL, Bø K, de Freitas LM, dela Ossa AMP, Bueno SM, et al. Do women have an accurate perception of their pelvic floor muscle contraction? A cross- sectional study.
Neurourol Urodyn. 2019;39(1):361-6. https://doi.org/10.1002/ nau.24214.

22. Andrade RL, Bø K, Antonio FI, et al. An education program about pelvic floor muscles improved women's knowledge but not pelvic floor muscle function, urinary incontinence or sexual function: a randomised trial. J Physiother. 2018;64(2):91-6. https://doi.org/ 10.1016/j.jphys.2018.02.010.

23. Vaz CT, Sampaio RF, Saltiel F, Figueiredo EM. Effectiveness of pelvic floor muscle training and bladder training for women with urinary incontinence in primary care: a pragmatic controlled trial. Braz J Phys Ther. 2019;23(2):116-24. https://doi.org/10.1016/j. bjpt.2019.01.007.

24. Barnes KL, Dunivan G, Jaramillo-Huff A, Krantz T, Thompson J, Jeppson P. Evaluation of smartphone pelvic floor exercise applications using standardized scoring system. Female Pelvic Med Reconstr Surg. 2018;25(4):328-35. https://doi.org/10.1097/SPV. 0000000000000563.

25. Hay-Smith J, Peebles L, Farmery D, Dean S, Grainger R. Appsolutely fabulous? - the quality of PFMT smartphone app content and design rated using the Mobile app rating scale, behaviour change taxonomy, and guidance for exercise prescription. Neurourol Urodyn 2019. https://www.ics.org/2019/abstract/547

26. Rao SSC, Valestin JA, Xiang X, Hamdy S, Bradley CS, Zimmerman MB. Home-based versus office-based biofeedback therapy for constipation with dyssynergic defecation: a randomised controlled trial. Lancet Gastroenterol Hepatol. 2018;3(11):768-77 http://www.sciencedirect.com/science/article/pii/ S2468125318302668.

27. Bernard S, Boucher S, McLean L, Moffet H. Mobile technologies for the conservative self-management of urinary incontinence: a systematic scoping review. Int Urogynecol J. 2020;31(6):1163-74.

28. Leme Nagib AB, Riccetto C, Martinho NM, et al. Use of mobile apps for controlling of the urinary incontinence: a systematic review. Neurourol Urodyn. 2020;39(4):1036-48.

29. Dantas LO, Barreto RPG, Ferreira CHJ. Digital physical therapy in the COVID-19 pandemic. Braz J Phys Ther 2020;S14133555(20)30402-0. https://doi.org/10.1016/j.bjpt.2020.04.006

30. Li C, Gong Y, Wang B. The efficacy of pelvic floor muscle training for pelvic organ prolapse: a systematic review and meta-analysis. Int Urogynecol J. 2016;27(7):981-92. https://doi.org/10.1007/ s00192-015-2846-y.

31. Mateus-Vasconcelos ECL, Brito LGO, Driusso P, Silva TD, Antônio FI, Ferreira CHJ. Effects of three interventions in facilitating voluntary pelvic floor muscle contraction in women: a randomized controlled trial. Braz J Phys Ther. 2018;22(5):391-9. https:// doi.org/10.1016/j.bjpt.2017.12.006.

Publisher's note Springer Nature remains neutral with regard to jurisdictional claims in published maps and institutional affiliations. 